\title{
Perivascular Spaces in Old Age: Assessment, Distribution, and Correlation with White Matter Hyperintensities
}

\author{
(D) Aaveskog, (D). Wang, (D). Bronge, (D).-O. Wahlund, and (D). Qiu
} O- $\equiv$

\begin{abstract}
BACKGROUND AND PURPOSE: The visual rating scales for perivascular spaces vary considerably. We sought to develop a new scale for visual assessment of perivascular spaces and to further describe their distribution and association with white matter hyperintensities in old age.
\end{abstract}

MATERIALS AND METHODS: This population-based study included 530 individuals who did not have dementia and were not institutionalized (age, $\geq 60$ years or older; mean age, 70.7 years; $58.9 \%$ women) who were living in central Stockholm, Sweden. A semiquantitative visual rating scale was developed to score the number and size of visible perivascular spaces in 7 brain regions in each hemisphere. $\mathrm{A}$ modified Scheltens visual rating scale was used to assess white matter hyperintensities.

RESULTS: The global scores for perivascular spaces ranged from 4-32 for number, 3-22 for size, and 7-54 for the combination of number and size. The weighted $\kappa$ statistics for the intra- and interrater reliability both were 0.77 . The global score for the number of perivascular spaces increased with advancing age $(P<.001)$. The scores for the number of perivascular spaces in the basal ganglia and subinsular regions were significantly correlated with the load of white matter hyperintensities, especially in lobar and deep white matter regions (partial correlation coefficients, $>0.223 ; P<.01)$.

CONCLUSIONS: The new visual rating scale for perivascular spaces shows excellent intra- and interrater reliability. The number of perivascular spaces globally and, especially, in the basal ganglia, is correlated with the load of lobar and deep white matter hyperintensities, supporting the view that perivascular spaces are a marker for cerebral small-vessel disease.

ABBREVIATIONS: ICD = International Classification of Diseases; PVH = periventricular hyperintensities; PVS = perivascular spaces; SNAC-K = Swedish National study on Aging and Care in Kungsholmen; STRIVE = STandards for Reporting Vascular changes on nEuroimaging; WMH = white matter hyperintensities

$T^{1}$ he perivascular spaces (PVS) are subpial virtual spaces between the adventitia of the vessel and the basal membrane of the glia limitans, involved in the lymphatic drainage of the brain. ${ }^{1,2}$ PVS are now easily identifiable in vivo, owing to improved MR imaging techniques. ${ }^{3}$ Since the late 1980s, different rating scales have been developed for the evaluation of PVS, depending on the purpose of the study. ${ }^{4-9}$ However, very few scales have simultaneously taken the number, size, and location of PVS

Received August 16, 2016; accepted after revision September 1, 2017.

From the Division of Radiology (A.L.), Department of Clinical Science, Intervention and Technology; Aging Research Center (R.W., C.Q.), Department of Neurobiology, Care Sciences and Society; and Department of Clinical Neuroscience (L.B.), Karolinska Institutet, Stockholm, Sweden; Department of Neuroradiology (A.L.), Karolinska University Hospital, Stockholm, Sweden; Aleris Diagnostics (L.B.), Sabbatsberg, Stockholm, Sweden; and Division of Clinical Geriatrics (L.-O.W.), Department of Neurobiology, Care Sciences and Society, Karolinska University Hospital at Huddinge, Stockholm, Sweden.

SNAC-K was supported by the Swedish Ministry of Health and Social Affairs, the Stockholm County Council, Stockholm Municipality, and the Swedish Research Council. This work was further supported, in part, by grants from the Swedish Research Council (grant Nos. 2015-02531 and 2016-06658); the Swedish Research Council for Health, Working Life and Welfare (grant No. 2014-01382); and the Karolinska Institutet, Stockholm, Sweden. into consideration. This is important because it remains unclear whether the number or the size of PVS is clinically relevant. Also, PVS in various anatomic regions of the brain may differ in risk factors, neuropathologies, and functional consequences. ${ }^{10,11}$ Thus, STandards for Reporting Vascular changes on nEuroimaging (STRIVE) have proposed to evaluate the number, size (maximum diameter), and anatomic location of PVS. ${ }^{12}$

PVS are common among elderly adults. Indeed, PVS are correlated with increasing age, ${ }^{4,9,13}$ though some earlier studies have shown equal distribution of PVS through a range of ages. ${ }^{14,15} \mathrm{In}$ addition, a sex difference in the distribution of PVS in the basal

\footnotetext{
Please address correspondence to Anna Laveskog, MD, Department of Neuroradiology, Karolinska University Hospital, S-171 76 Stockholm, Sweden; e-mail: anna.laveskog@sll.se; Rui Wang, PhD, Aging Research Center, Karolinska Institutet, Gävlegatan 16, 11330 Stockholm, Sweden; e-mail: rui.wang@ki.se; or Chengxuan Qiu, MD, Aging Research Center, Karolinska Institutet, Gävlegatan 16, 11330 Stockholm, Sweden; e-mail: chengxuan.qiu@ki.se

- Indicates open access to non-subscribers at www.ajnr.org

三 Indicates article with supplemental on-line tables.

Indicates article with supplemental on-line photo

http://dx.doi.org/10.3174/ajnr.A5455
} 

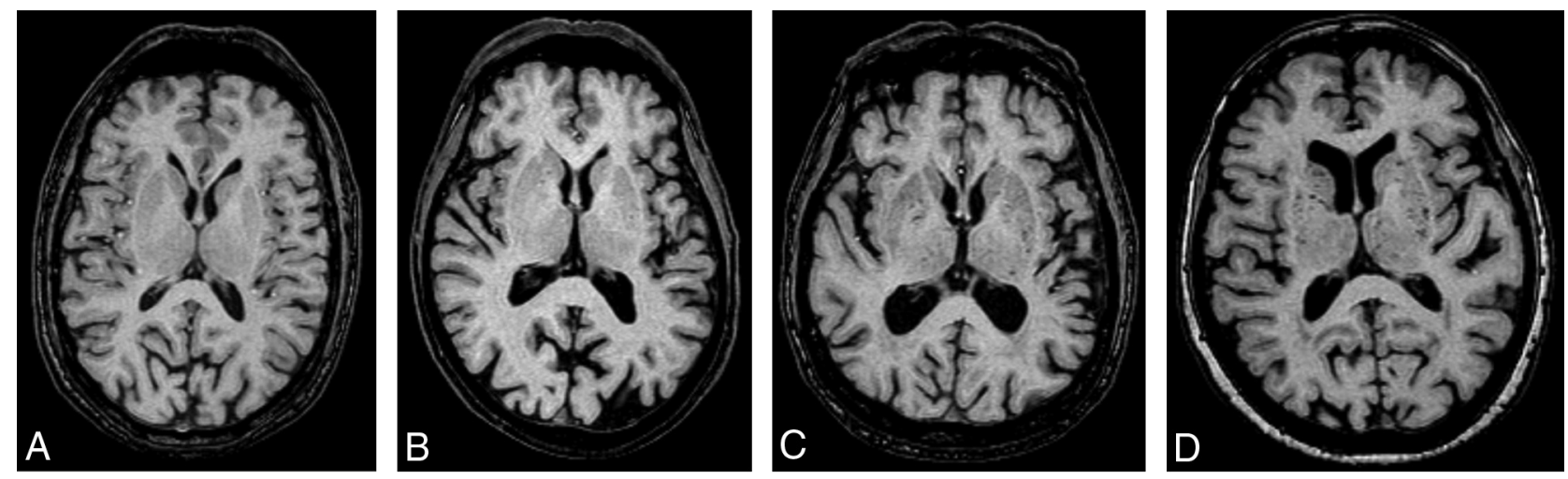

FIG 1. Sample images of grades $0-3$ for the number of perivascular spaces bilaterally in the basal ganglia medial to the external capsule. The images indicate grade $0(A)$, grade $1(B)$, grade $2(C)$, and grade $3(D)$ in the basal ganglia. Note that the perivascular spaces contributing to the score might be in another level and thus not visible in the sample images.

ganglia has been reported, with men having more PVS than women. ${ }^{11,13,16}$ The most common etiologies proposed for the widening of the originally virtual PVS to become visible on MR imaging are hypertension ${ }^{13,17}$ and amyloid deposition, at least in patients with Alzheimer disease. ${ }^{18}$ Some studies have suggested that PVS are associated with cognitive decline and dementia. ${ }^{6,13}$

In the past decade, studying PVS as an imaging marker for cerebral small-vessel disease represents one of the research frontiers in brain aging. ${ }^{8}$ Some studies have reported that PVS are correlated with the load of both white matter hyperintensities (WMH) and periventricular hyperintensities (PVH) independent of age and vascular risk factors, ${ }^{19}$ suggesting that PVS may be an imaging marker for cerebral small-vessel disease. However, it has been unclear whether PVS in various anatomic regions are differentially associated with global and regional WMH because PVS in different regions may have different origins and etiopathologies. ${ }^{10}$

Here we present data from a population-based MR imaging study within the Swedish National study on Aging and Care in Kungsholmen (SNAC-K) in central Stockholm, Sweden. ${ }^{20}$ Within this study, we have the unique opportunity to describe a new visual rating scale for PVS by considering the number, size, and location of PVS. Furthermore, we sought to investigate the age- and sex-specific distribution of PVS in older individuals and to explore whether global and regional PVS are associated with the load of global and regional WMH.

\section{MATERIALS AND METHODS \\ Study Participants}

Participants were derived from the SNAC-K study, a multidisciplinary study of aging and health, as fully reported elsewhere. ${ }^{20}$ Briefly, the SNAC-K study included 4 younger cohorts with 6 -year intervals $(60,66,72$, and 78 years of age $)$ and 7 older cohorts with 3-year intervals $(81,84,87,90,93,96$, and 99 and older years of age). Of all 4590 persons who were eligible to participate in SNAC-K, 3363 (73.3\%) were eventually examined at baseline (March 2001 to June 2004). From September 2001 to October 2003, five hundred fifty-five SNAC-K participants who were not institutionalized or disabled and who did not have dementia also underwent brain MR imaging examinations. ${ }^{20}$ Of these, 2 persons did not complete the entire examination owing to claustrophobia, 4 had suboptimal MR imaging because of motion artifacts, 2 were excluded because of large meningiomas, and images for 17 persons were lost due to technical problems. Therefore, 530 subjects were included in this study. All the participants had a Mini-Mental State Examination score of $\geq 21$ (mean score, 29). Fifteen brain regions in 6 subjects were impossible to assess because of large infarcts. Therefore, these regions, but not other regions in these subjects, were excluded from our analysis.

SNAC-K was approved by the regional ethics review board in Stockholm, Sweden. Written informed consent was collected from all participants in the SNAC-K MR imaging sample.

\section{MR Imaging Acquisition}

All participants were scanned on an Intera 1.5T system (Philips Healthcare, Best, the Netherlands). For the visual assessment of PVS, a T1-weighted sequence (MPRAGE; TR, $15 \mathrm{~ms}$; TE, $7 \mathrm{~ms}$; flip angle, $15^{\circ}$ ) consisting of one hundred fifty $1.5-\mathrm{mm}$ axial sequential images without angulation; a FLAIR sequence (TR, 6000 ms; TI, 1900 ms; TE, 100 ms; echo-train length, 21; flip angle 90) of twenty $5-\mathrm{mm}$ sequential images, with a gap of $1 \mathrm{~mm}$, angled to the subcallosal line; and a proton-density/T2-weighted sequence (TR, 3995 ms; TE, 18/90 ms; echo-train length, 6; flip angle, 90) of sixty $3-\mathrm{mm}$ sequential images without a gap or angulation were used. All images were reviewed on a clinical PACS system.

\section{Visual Assessment of PVS}

We constructed a visual rating scale while considering the number, size, and location of PVS. The assessment was performed separately for each hemisphere, and the different locations of the brain were chosen on the basis of the regions used in earlier studies. ${ }^{6,7,21}$ In each region of both hemispheres, the number and maximum diameter of PVS were scored and recorded separately. The scores for both the number and size were then summed up to obtain a global semiquantitative measurement of visible PVS, with a maximum score of 84 .

The brain regions scored were the cerebellum, mesencephalon (including the upper part of pons), hippocampus, subinsular territory (external capsule, claustrum, and extreme capsule), basal ganglia (medial to external capsule, including the thalamus and internal capsule), frontal lobe, and parieto-occipital lobe. Visible PVS in each region were counted and then scored as 0 (no visible PVS), 1 (1-5 PVS), 2 (6-10 PVS), or 3 (>10 PVS) (Fig 1 and 

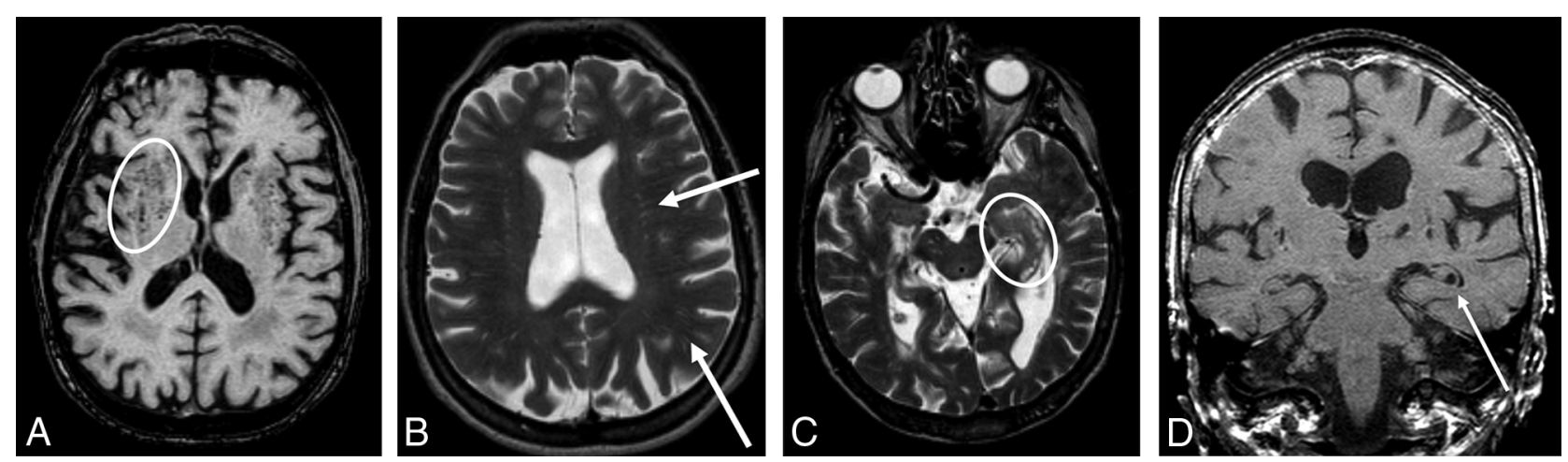

FIG 2. Sample images of PVS with different grades. $A$ and $B$, Grade 3 in number and grade 1 in size of PVS in the subinsular, basal ganglia, frontal, and parietal regions. There are $>10$ PVS in each region, but none of the PVS are wider than $2 \mathrm{~mm}$. In the basal ganglia, PVS are seen as dots (oval) because the image is acquired cross-sectional to the vessels ( $A$, axial TT), while PVS are linear in the frontal and parietal lobe (arrows), where the vessels are in a plane with the image (B, axial T2). $C$, Grade 3 in number and grade 1 in size in the left hippocampus (oval, axial T2). PVS in the hippocampus are really hippocampal sulcus remnants, which can be clearly seen in $D$ (arrow, coronal TT).

On-line Table 1). When we measured the diameter of obliquely imaged PVS, the smallest diameter of each periventricular space was used. The maximum diameter of PVS was scored as 0 (no visible PVS), 1 (1-2 mm), 2 (3-4 mm), and 3 (>4 mm). All images were assessed by an experienced clinical neuroradiologist (A.L.). Six months after the initial reading, MR images of 20 randomly selected subjects were re-evaluated for PVS, which yielded a weighted $\kappa$ statistic of 0.77 (intrarater reliability). In addition, MR images of 18 subjects were evaluated for PVS by an experienced general radiologist (L.B.); this evaluation yielded a weighted $\kappa$ statistic of 0.77 (interrater reliability).

We used the T1-weighted 3D volume images to count the total number of PVS in the basal ganglia and subinsular region and followed them through the sections, which facilitated separating PVS from lacunes and choroidal fissure cysts and avoided counting the same space twice. With T1, instead of T2, images for the basal ganglia also diminished the disturbance from WMH common in this region that might be confused with PVS. The PVS in the frontal and parietal lobes were more easily assessed on the axial T2 images as well as hippocampal sulcus remnants and PVS in cerebellum and mesencephalon (Fig 2). The FLAIR images were used to distinguish PVS from lacunes. The changes were considered to constitute lacunes if they were surrounded by a high signal on FLAIR images or if they did not have a rounded elongated shape. However, because the scale is based on counting the number and measuring the diameters of PVS, different sequences with thin sections might be used.

\section{Visual Assessment of WMH and PVH}

WMH were defined as hyperintense signal on FLAIR images. The load of WMH was rated using a modified Scheltens rating scale (On-line Table 2). ${ }^{22}$ The rating scale is the same as the original Scheltens scale, but the regionalization is different. First, we rated the 2 hemispheres separately. Second, due to very few white matter changes in the thalami and the cognitive importance of the external capsule, the basal ganglia and thalami were divided into subinsular region (external capsule, claustrum, and extreme capsule) and basal ganglia (medial to external capsule, including the thalamus and internal capsule). Hyperintense changes in the basal ganglia and thalami were included in the $\mathrm{WMH}$, as previously suggested. ${ }^{23}$ Finally, we combined the parietal and occipital lobes into 1 region due to the low number of WMH in the occipital lobe. PVH were rated according to the original Scheltens scale. ${ }^{22}$

All assessments of WMH were completed by a clinical neuroradiologist (A.L.) without knowledge of subjects' clinical characteristics. The intrarater reliability for $\mathrm{WMH}$ was good, with the weighted $\kappa$ statistic of 0.67 .

\section{Assessment of Cardiovascular Health}

Data on demographics (eg, age, sex, and education) and cardiovascular risk factors and disorders were collected through face-to-face interviews, clinical examinations, laboratory tests, and patient registers. ${ }^{20}$ Hypertension was defined as arterial blood pressure of $\geq 140 / 90 \mathrm{~mm} \mathrm{Hg}$ or current use of antihypertensive agents. Obesity was defined as a body mass index of $\geq 30 \mathrm{~kg} / \mathrm{m}^{2}$. High total cholesterol was defined as a total serum cholesterol of $\geq 6.22 \mathrm{mmol} / \mathrm{L}$ or use of cholesterol-lowing agents. Diabetes was defined as having a selfreported history of diabetes, a record of diabetes in the patient register, use of hypoglycemic agents, or an HbAlc of $\geq 5.4 \%$.

A history of heart disease was ascertained according to medical records in the Stockholm patient register. We included coronary heart disease (International Classification of Diseases, Ninth Revision [ICD-9] codes 410-414; ICD-10 codes I20-I25); heart failure (ICD- 9 code 428 and ICD-10 code I50); and atrial fibrillation (ICD-9 code 427.8 and ICD-10 code I48).

\section{Statistical Analysis}

Demographic characteristics of study participants by sex were compared using the $t$ test for continuous variables and the $\chi^{2}$ test for categoric variables. We used a scatterplot to show the distribution of global PVS scores by age, and the correlations of PVS scores with age were explored with the general linear regression model. To demonstrate the distribution of the regional PVS load, we presented the number and proportion of subjects according to the scores of PVS in each brain region for both hemispheres. Partial correlation was used to investigate the correlation between PVS scores and WMH scores globally and by regions, controlling for age, sex, and education. STATA, Version 12.0 for Windows (StataCorp, College Station, Texas) was used for all analyses. 
Table 1: Demographic and clinical characteristics of study participants by sex

\begin{tabular}{lcccc}
\hline \multicolumn{1}{c}{ Characteristics } & Total Sample & Men & Women & $\boldsymbol{P}$ Value $^{\mathbf{a}}$ \\
\hline No. of subjects & 530 & 218 & 312 & \\
Age (yr) (No.) (\%) & & & & \\
60 & $141(26.6)$ & $66(30.3)$ & $75(24.0)$ & \\
66 & $123(23.2)$ & $51(23.4)$ & $72(23.1)$ & \\
72 & $84(15.8)$ & $30(13.8)$ & $54(17.3)$ & \\
78 & $73(13.8)$ & $29(13.3)$ & $44(14.1)$ & \\
81 & $49(9.2)$ & $18(8.3)$ & $31(9.9)$ & \\
84 & $26(4.9)$ & $9(4.1)$ & $17(5.4)$ & \\
87 & $18(3.4)$ & $9(4.1)$ & $9(2.9)$ & \\
90 or older & $16(3.0)$ & $6(2.8)$ & $10(3.2)$ & .72 \\
Age (yr), mean (SD) & $70.7(9.1)$ & $70.1(9.2)$ & $71.1(9.0)$ & .20 \\
Education (No.) (\%) & & & & \\
Primary school & $67(12.6)$ & $26(11.9)$ & $41(13.1)$ & \\
Middle school & $246(46.4)$ & $78(35.8)$ & $168(53.8)$ & \\
University & $217(40.9)$ & $114(52.3)$ & $103(33.0)$ & $<.01$ \\
Hypertension (No.) (\%) & $383(72.3)$ & $165(75.7)$ & $218(69.9)$ & .14 \\
Diabetes (No.) (\%) & $41(7.7)$ & $25(11.5)$ & $16(5.1)$ & $<.01$ \\
High cholesterol (No.) (\%) & $295(56.4)$ & $118(55.7)$ & $177(56.9)$ & .78 \\
Obesity (No.) (\%) & $72(13.6)$ & $30(13.8)$ & $42(13.5)$ & .93 \\
Atrial fibrillation (No.) (\%) & $11(2.1)$ & $7(3.2)$ & $4(1.3)$ & .13 \\
Coronary heart disease (No.) (\%) & $53(10.0)$ & $30(13.8)$ & $23(7.4)$ & .02 \\
Heart failure (No.) (\%) & $12(2.3)$ & $5(2.3)$ & $7(2.2)$ & .97 \\
PVH (median) (IQR) & $8(6-9)$ & $8(6-10)$ & $8(6-9)$ & .56 \\
WMH (median) (IQR) & $16(5-29)$ & $16(4-26)$ & $16(6-30)$ & .56 \\
\hline
\end{tabular}

Note:-IQR indicates interquartile range.

a $P$ value was for the test of sex differences.

\section{RESULTS}

\section{Characteristics of Study Participants}

Table 1 shows the demographic and clinical characteristics of the 530 participants by sex. The mean age of the sample was $70.7 \pm$ 9.1 years, and $58.9 \%$ were women. Men were more likely than women to have a higher level of education $(P<.01)$, diabetes $(P<.01)$, and coronary heart disease $(P=.02)$. There were no significant sex differences in the mean age, prevalence of hypertension, high total cholesterol, obesity, atrial fibrillation, and heart failure or in the severity of WMH and PVH $(P>.10)$ (Table 1).

\section{Distribution of Global PVS}

The means of the global score for the number of PVS were $18.7 \pm$ 5.2 (range, $4-32$ ); $10.7 \pm 2.6$ for the size of PVS (range, $3-22$ ); and $29.3 \pm 7.4$ for combining the number and size of PVS (range, 7-54). The PVS total score shows approximately normal distribution (On-line Figure). Large PVS ( $>4 \mathrm{~mm}$ ) were detected in only 6 individuals $(1.1 \%)$ of all the 530 participants or in 29 regions $(0.4 \%)$ of a total number of 7405 brain regions (each person having 7 regions in each hemisphere).

The global score for the combination of both the number and size of PVS increased with advancing age $(\beta$ coefficient $=0.218$, $P<.01)$ as did the global score for the number of PVS ( $\beta$ coefficient $=0.162, P<.01$ ). The global score for PVS size did not vary substantially with age, though the correlation was statistically significant $(\beta$ coefficient $=0.057, P<.01)$ (Fig 3). There was no significant sex difference in the distribution of global scores for either the number or size of PVS.

\section{Distribution Patterns of Regional PVS}

Around $60 \%$ of the subjects showed $>10$ PVS in the frontal and parieto-occipital lobes; $\sim 45 \%$ showed $6-10$ PVS in the basal gan- glia; $>50 \%$ showed $1-5$ PVS in the subinsular, hippocampus, or mesencephalon regions; and $>85 \%$ of the subjects did not have any PVS in the cerebellum region (Table 2 ).

For distribution of regional PVS by size, $>80 \%$ of the subjects had a PVS size of $1-2 \mathrm{~mm}$ in the frontal lobe, parietooccipital lobe, or basal ganglia. The most common region for having PVS of $\geq 3$ $\mathrm{mm}$ was the basal ganglia $(\sim 15 \%$ in both hemispheres). The distribution of PVS by both number and size appeared to be symmetric in both hemispheres.

\section{Correlation between WMH and PVS}

After we controlled for demographics, the global scores for the number, size, and both the number and size of PVS were all positively correlated with the WMH score in lobar and deep white matter areas $(P<.05$ or $P<.01)$, whereas there was no significant correlation between global PVS and PVH scores (Table 3 ).

Higher scores for the number of PVS in the basal ganglia and subinsular areas were significantly associated with a higher global WMH score and a higher WMH score in the lobar, deep, and periventricular areas. Furthermore, a higher score for the number or size of PVS in the hippocampus was significantly associated with higher WMH scores in lobar areas. Finally, PVS scores in the frontal lobe, parieto-occipital lobe, mesencephalon, and cerebellum were largely not correlated with WMH scores.

\section{DISCUSSION}

In this population-based study of older adults, we have developed a new rating scale for visual assessment of PVS, considering the number, size, and location, which meets the requirements of STRIVE, the neuroimaging standards for research into cerebral small-vessel disease. ${ }^{12}$ With this scale, we show evidence that PVS are an imaging marker for cerebral small-vessel disease and that the number, rather than the size, might be a more important measure for PVS. In addition, using this scale, we will be able to investigate different risk factors for and functional consequences of PVS by various brain areas.

When we started studying PVS, there were several scales for visual assessment available, even though few scales have been used in $>1$ study, probably because most of these scales had great benefits, but also drawbacks. Most of the previous scales were only concerned with the PVS number and used a fixed diameter of $2^{24}$ or $3 \mathrm{~mm}^{19}$ to separate PVS from lacunes, even though PVS can sometimes grow very large. ${ }^{25}$ For instance, one scale measured the mean diameters of all PVS in 1 axial section at the level of the cella media of the lateral ventricles, which means that the scale mainly evaluated the frontal and parietal white matter. ${ }^{5}$ This scale left out the basal ganglia, where many PVS are located. In another scale, the PVS both along the lenticulostriate arteries (in the basal gan- 


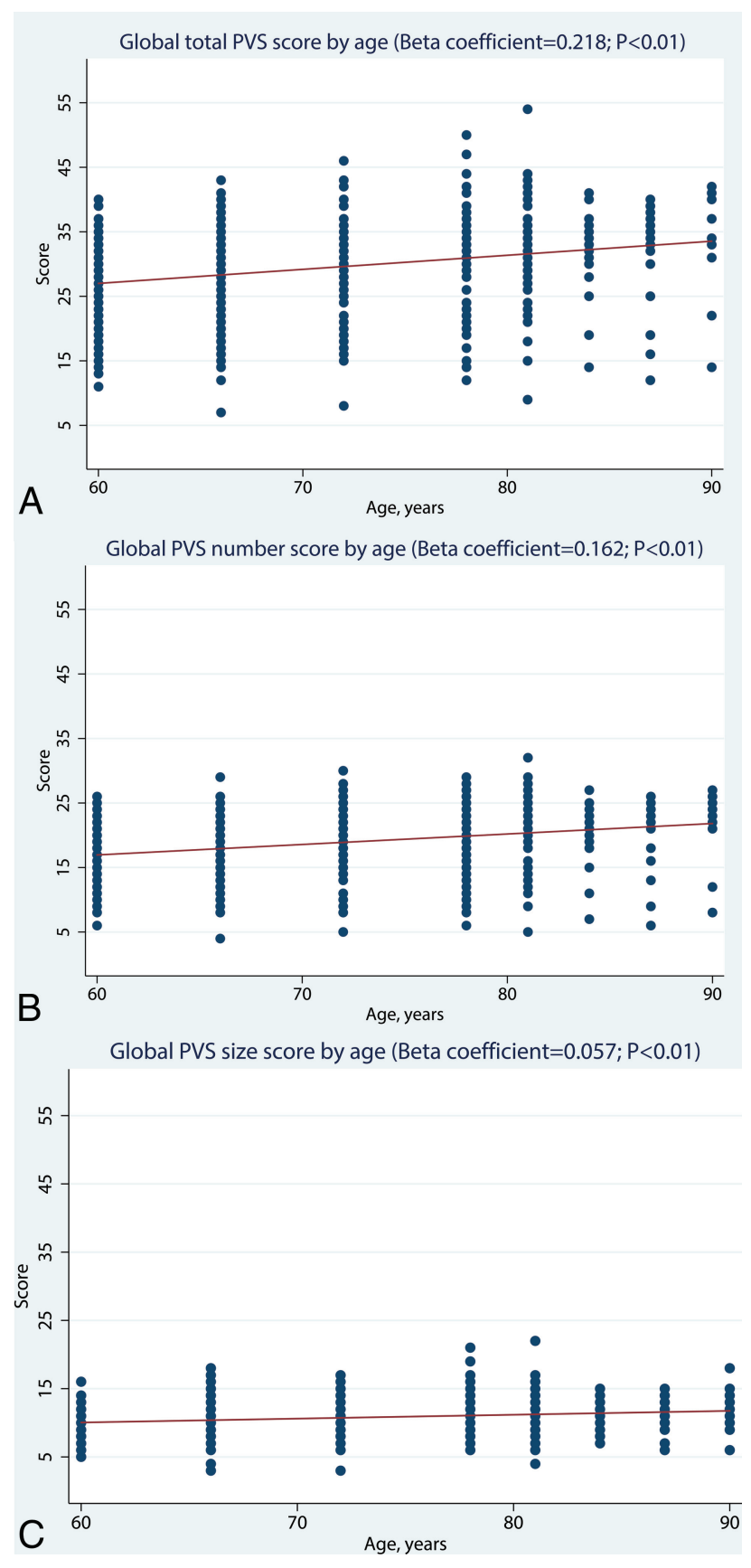

FIG 3. Age-specific distribution of global scores for the number, size, and a combination of both number and size of PVS. Note that there are 3 subjects with missing values in the global PVS score for size.

glia) and in the high convexities were assessed. ${ }^{4}$ The scale was based on the width of PVS but was also given 1 point if there were $>4$ PVS in each region. Thus, the scale did not really separate the diameter from the number of PVS. The third scale rated the number of PVS only in the most affected hemisphere but combined the basal ganglia with the centrum semiovale and rated only the hippocampus separately. ${ }^{6}$ Thus, this scale could not differentiate PVS in the basal ganglia from those in the centrum semiovale. This drawback was overcome in a later study. ${ }^{19}$

The scale by Patankar et $\mathrm{al}^{7}$ rated the number of PVS in 4 different regions, which makes this scale very appealing; thus, it has been used by other groups. ${ }^{26,27}$ However, this scale has a great disadvantage in a complicated grading, where each region has a different grading scale with 2 different schemes for the basal ganglia. Because we aimed to develop a rating scale that was easy to use, we decided to use the grades $0-3$ for both the diameter and number of PVS. Our rating scale is in line with the requirements of the STRIVE in terms of rating both the number and size in different locations. ${ }^{12}$

We found a slight increase in the global score for PVS number with advancing age, which is in accordance with most other studies. ${ }^{4,8,9,13,28-31}$ Our study sample was relatively healthy, ${ }^{20}$ which might partly explain a smaller increase with age than expected, because PVS are presumably correlated with the degree of cerebral small-vessel disease and amyloid deposition with increasing age. ${ }^{17,18}$ We did not find a sex difference in the distribution of PVS, even though it has been reported in earlier studies ${ }^{11,13,16}$; notably, one of these studies comprised a very large population-based sample ( $n=\sim 1800)$, in which men were found to have more PVS in the basal ganglia than women, ${ }^{13}$ while another large-scale study of patients with ischemic stroke $(n=$ 1090) found that men had more PVS in the white matter than women, but there was no sex difference in the distribution of PVS in the basal ganglia. ${ }^{11}$ The discrepancies in the findings may be partly attributable to the differences in characteristics of the study populations.

In our sample, we found very few PVS $>4 \mathrm{~mm}(1.1 \%$ of all subjects or $0.4 \%$ of all the examined brain regions), which is in accordance with the traditional view that PVS should be $<5$ $\mathrm{mm} .{ }^{32}$ In addition, our data showed rather limited variation in the size of PVS by age. This appears to be contradictory to a clinically based study, which showed a strong correlation of the width of the PVS with increasing age. ${ }^{4}$ However, because the scale in that study yielded a higher score if there were many PVS, it might have reflected an increasing number, rather than size, of PVS with age. Given that the width of PVS has been correlated with advancing age, ${ }^{4}$ it might still be important to also include the diameter when assessing PVS.

A higher global PVS score was correlated with a greater burden of WMH in lobar regions and the basal ganglia, which supports the view that PVS, along with WMH, are markers for cerebral small-vessel disease. ${ }^{9,19,33}$ More interesting, we found a correlation between the PVS score in the basal ganglia and WMH load not only in the same region but also in lobar areas, while we did not find any correlation between the lobar PVS score and WMH load either in the lobar areas or in the basal ganglia. This is in line with the view that PVS, especially in the basal ganglia, are caused mainly by hypertensive arteriopathy, like WMH, while lobar PVS are mainly due to cerebral amyloid angiopathy or amyloid accumulation in the brain with normal aging. ${ }^{10,30}$

A major strength of our study is the large sample from the general population. To date, only very few studies have had such a large sample of community dwellers. ${ }^{4,13,29}$ In addition, because our scale is based on counting the number and measuring the diameter of PVS, it can be used on different MR imaging sequences, as opposed to the visual impression-based rating scales. Furthermore, the regionalization in the assessments of both PVS and $\mathrm{WMH}$ is of relevance when studying their correlations with vascular risk factors and cognitive aging. 
Table 2: Distribution of regional perivascular spaces by number and size ${ }^{\mathrm{a}}$

\begin{tabular}{|c|c|c|c|c|c|c|c|c|}
\hline \multirow{2}{*}{$\begin{array}{l}\text { Hemispheres } \\
\text { and Regions }\end{array}$} & \multicolumn{4}{|c|}{ No. of Perivascular Spaces } & \multicolumn{4}{|c|}{$\begin{array}{l}\text { Size (mm in Diameter) } \\
\text { of Perivascular Spaces }\end{array}$} \\
\hline & None & $1-5$ & $6-10$ & $>10$ & None & $1-2$ & $3-4$ & $>4$ \\
\hline \multicolumn{9}{|l|}{ Left } \\
\hline Frontal & $32(6.0)$ & $70(13.2)$ & $107(20.2)$ & $321(60.6)$ & $32(6.0)$ & $473(89.3)$ & $25(4.7)$ & 0 \\
\hline Parieto-occipital & $25(4.7)$ & $68(12.8)$ & $116(21.9)$ & $321(60.6)$ & $25(4.7)$ & $486(91.7)$ & $16(3.0)$ & $3(0.6)$ \\
\hline Basal ganglia & 0 & $85(16.0)$ & $240(45.3)$ & $205(38.7)$ & 0 & $443(83.6)$ & $80(15.1)$ & $7(1.3)$ \\
\hline Subinsular & $128(24.2)$ & $277(52.3)$ & $107(20.2)$ & $18(3.4)$ & $128(24.2)$ & $370(69.9)$ & $30(5.7)$ & $1(0.2)$ \\
\hline Hippocampus & $156(29.4)$ & $328(61.9)$ & $46(8.7)$ & 0 & $156(29.4)$ & 348 (65.7) & $26(4.9)$ & 0 \\
\hline Mesencephalon & $255(48.1)$ & $274(51.9)$ & 0 & 0 & $254(47.9)$ & $273(51.5)$ & $3(0.6)$ & 0 \\
\hline Cerebellum & $456(86.0)$ & $74(14.0)$ & 0 & 0 & $456(86.0)$ & 74 (14.0) & 0 & 0 \\
\hline \multicolumn{9}{|l|}{ Right } \\
\hline Frontal & $28(5.3)$ & $64(12.1)$ & 94 (17.7) & $344(64.9)$ & $28(5.3)$ & 474 (89.4) & $27(5.1)$ & $1(0.2)$ \\
\hline Parieto-occipital & $29(5.5)$ & 79 (14.9) & $106(20.0)$ & $316(59.6)$ & $29(5.5)$ & 487 (91.9) & $14(2.6)$ & 0 \\
\hline Basal ganglia & $1(0.2)$ & 103 (19.4) & $231(43.6)$ & $195(36.8)$ & $1(0.2)$ & $452(85.3)$ & 63 (11.9) & $14(2.6)$ \\
\hline Subinsular & $131(24.7)$ & $280(52.8)$ & 103 (19.4) & $16(3.0)$ & $131(24.7)$ & $369(69.6)$ & $29(5.5)$ & $1(0.2)$ \\
\hline Hippocampus & $210(39.6)$ & $284(53.6)$ & $35(6.6)$ & $1(0.2)$ & $209(39.5)$ & $292(55.2)$ & $26(4.9)$ & $2(0.4)$ \\
\hline Mesencephalon & $263(49.6)$ & $265(50.0)$ & $2(0.4)$ & 0 & $262(49.5)$ & $263(49.7)$ & $4(0.8)$ & 0 \\
\hline Cerebellum & $462(87.2)$ & $68(12.8)$ & 0 & 0 & $463(87.4)$ & $67(12.6)$ & 0 & 0 \\
\hline
\end{tabular}

a Data are No. (\%).
${ }^{b}$ There were missing values of perivascular space size in 1 subject in the mesencephalon (right hemisphere), 1 in the hippocampus (right hemisphere), and 1 in the basal ganglia (left hemisphere).

Table 3: Partial correlation coefficients between PVS and WMH scores ${ }^{\mathrm{a}}$

\begin{tabular}{|c|c|c|c|c|}
\hline \multirow[b]{2}{*}{$\begin{array}{c}\text { Global and Regional } \\
\text { PVS Scores }\end{array}$} & \multicolumn{4}{|c|}{ WMH Scores } \\
\hline & Global & $\begin{array}{l}\text { Lobar White } \\
\text { Matter Areas }\end{array}$ & $\begin{array}{l}\text { Deep White } \\
\text { Matter Areas }\end{array}$ & $\begin{array}{l}\text { Periventricular } \\
\text { Regions }^{\mathrm{b}}\end{array}$ \\
\hline \multicolumn{5}{|l|}{ PVS score, total } \\
\hline Global region & $0.204^{c}$ & $0.182^{c}$ & $0.171^{c}$ & 0.066 \\
\hline Frontal region & 0.040 & 0.048 & 0.011 & 0.007 \\
\hline Parieto-occipital region & 0.028 & 0.017 & 0.028 & 0.031 \\
\hline Basal ganglia & $0.318^{c}$ & $0.256^{\mathrm{c}}$ & $0.292^{c}$ & $0.160^{c}$ \\
\hline Subinsular region & $0.228^{c}$ & $0.206^{c}$ & $0.173^{c}$ & $0.101^{\mathrm{d}}$ \\
\hline Hippocampus & $0.127^{c}$ & $0.137^{c}$ & 0.072 & 0.021 \\
\hline Mesencephalon & 0.044 & 0.044 & 0.073 & -0.077 \\
\hline Cerebellum & 0.071 & 0.034 & $0.110^{d}$ & 0.039 \\
\hline \multicolumn{5}{|l|}{ PVS score (No.) } \\
\hline Global region & $0.206^{c}$ & $0.177^{c}$ & $0.183^{c}$ & 0.074 \\
\hline Frontal region & 0.034 & 0.039 & 0.015 & 0.001 \\
\hline Parieto-occipital region & 0.002 & -0.013 & 0.018 & 0.022 \\
\hline Basal ganglia & $0.355^{c}$ & $0.295^{c}$ & $0.303^{c}$ & $0.194^{c}$ \\
\hline Subinsular region & $0.263^{c}$ & $0.223^{c}$ & $0.233^{c}$ & $0.111^{\mathrm{d}}$ \\
\hline Hippocampus & $0.101^{d}$ & $0.117^{c}$ & 0.051 & -0.004 \\
\hline Mesencephalon & 0.041 & 0.044 & 0.067 & -0.081 \\
\hline Cerebellum & 0.071 & 0.034 & $0.11^{\mathrm{d}}$ & 0.037 \\
\hline \multicolumn{5}{|l|}{ PVS score (size) } \\
\hline Global region & $0.184^{c}$ & $0.177^{c}$ & $0.140^{c}$ & 0.042 \\
\hline Frontal region & 0.046 & 0.061 & -0.004 & 0.023 \\
\hline Parieto-occipital region & $0.109^{d}$ & $0.113^{d}$ & 0.052 & 0.053 \\
\hline Basal ganglia & 0.070 & 0.042 & $0.107^{d}$ & 0.008 \\
\hline Subinsular region & $0.143^{d}$ & $0.152^{d}$ & 0.059 & 0.073 \\
\hline Hippocampus & $0.151^{c}$ & $0.154^{c}$ & $0.092^{d}$ & 0.043 \\
\hline Mesencephalon & 0.002 & 0.012 & 0.029 & $-0.096^{\mathrm{d}}$ \\
\hline Cerebellum & 0.071 & 0.033 & 0.111 & 0.041 \\
\hline
\end{tabular}

a The correlation coefficients were adjusted for age, sex, and education.

${ }^{b}$ Lobar white matter areas include the frontal, parieto-occipital, and temporal lobes in both hemispheres; deep white matter areas include the basal ganglia, capsular, thalami, pons, and mesencephalon; and periventricular areas include the frontal, lateral, and occipital periventricular areas.

${ }^{c} P<.01$.

$\mathrm{d} p<.05$.

Our study also has limitations. First, we used diameter to measure the severity of PVS, which may be less precise than the direct measurement of PVS volume. Indeed, semiautomatic approaches for assessing PVS volume are being developed, ${ }^{16}$ though current volumetric methods do not cover the entire brain or work as fast as visual rating approaches. ${ }^{34,35}$ Second, our scale includes 2 parameters (number and size) of PVS in 14 regions, which is rather time-consuming (on average 6 minutes per examination). This means that our study has an advantage for research purposes. For visual assessment of several imaging markers (eg, WMH, microbleeds, and brain atrophy) or for clinical use, however, the scale of Potter et $\mathrm{al}^{9}$ may be easier to use for rating PVS in the most important regions such as the basal ganglia and centrum semiovale.

\section{CONCLUSIONS}

We describe a new semiquantitative scale for visual assessment of PVS that considers the number and diameter of PVS as well as location. This rating scale has excellent intra- and interrater reliability. Using this scale, we found that the global number of PVS increased with age and correlated with increasing load of WMH in lobar and deep white matter regions. This rating scale will be useful for future research on PVS, especially regarding risk factors and functional consequences of PVS by region. We plan to further investigate vascular risk factors and cognitive decline associated with global and regional PVS. A potential implication of linking global and regional PVS to functional outcomes is that the presence of visible PVS may be important to include in clinical reports on brain MR imaging examinations in the future, as we currently do for WMH in our clinical reports. 


\section{ACKNOWLEDGMENTS}

We are grateful to all the SNAC-K participants and to our colleagues in the SNAC-K group for their collaboration in data collection and management. We thank Dr Heather Martin from the Department of Neuroradiology, Karolinska University Hospital, Stockholm, Sweden, for careful editing of our manuscript.

Disclosures: Rui Wang — RELATED: Grant: The Swedish Ministry of Health and Social Affairs, the participating County Councils and Municipalities, and the Swedish Research Council. Chengxuan Qui-RELATED: Grant: The Swedish Ministry of Health and Social Affairs, the participating County Councils and Municipalities, the Swedish Research Council, and the Swedish Research Council for Health, Working Life and Welfare.* *Money paid to the institution.

\section{REFERENCES}

1. Esiri MM, Gay D. Immunological and neuropathological significance of the Virchow-Robin space. J Neurol Sci 1990;100:3-8 CrossRef Medline

2. Weller RO, Subash M, Preston SD, et al. Perivascular drainage of amyloid-beta peptides from the brain and its failure in cerebral amyloid angiopathy and Alzheimer's disease. Brain Path 2008;18: 253-66 CrossRef Medline

3. Groeschel S, Chong WK, Surtees R, et al. Virchow-Robin spaces on magnetic resonance images: normative data, their dilatation, and a review of the literature. Neuroradiology 2006;48:745-54 CrossRef Medline

4. Heier LA, Bauer CJ, Schwartz L, et al. Large Virchow-Robin spaces: MR-clinical correlation. AJNR Am J Neuroradiol 1989;10:929-36 Medline

5. Hiroki M, Miyashita K. Linear hyperintensity objects on magnetic resonance imaging related to hypertension. Cerebrovasc Dis 2001; 11:164-68 CrossRef Medline

6. Maclullich AM, Wardlaw JM, Ferguson KJ, et al. Enlarged perivascular spaces are associated with cognitive function in healthy elderly men. J Neurol Neurosurg Psychiatry 2004;75:1519-23 CrossRef Medline

7. Patankar TF, Mitra D, Varma A, et al. Dilatation of the VirchowRobin space is a sensitive indicator of cerebral microvascular disease: study in elderly patients with dementia. AJNR Am J Neuroradiol 2005;26:1512-20 Medline

8. Rouhl RP, van Oostenbrugge RJ, Knottnerus IL, et al. VirchowRobin spaces relate to cerebral small vessel disease severity. J Neurol 2008;255:692-96 CrossRef Medline

9. Potter GM, Chappell FM, Morris Z, et al. Cerebral perivascular spaces visible on magnetic resonance imaging: development of a qualitative rating scale and its observer reliability. Cerebrovasc Dis 2015;39:224-31 CrossRef Medline

10. Charidimou A, Jaunmuktane Z, Baron JC, et al. White matter perivascular spaces: an MRI marker in pathology-proven cerebral amyloid angiopathy? Neurology 2014;82:57-62 CrossRef Medline

11. Zhang C, Chen Q, Wang Y, et al; Chinese IntraCranial AtheroSclerosis (CICAS) Study Group. Risk factors of dilated Virchow-Robin spaces are different in various brain regions. PLoS One 2014;9: e105505 CrossRef Medline

12. Wardlaw JM, Smith EE, Biessels GJ, et al; STandards for ReportIng Vascular changes on nEuroimaging (STRIVE v1). Neuroimaging standards for research into small vessel disease and its contribution to ageing and neurodegeneration. Lancet Neurol 2013;12:822-38 CrossRef Medline

13. Zhu YC, Dufouil C, Soumare A, et al. High degree of dilated Virchow-Robin spaces on MRI is associated with increased risk of dementia. J Alzheimers Dis 2010;22:663-72 CrossRef Medline

14. Jungreis CA, Kanal E, Hirsch WL, et al. Normal perivascular spaces mimicking lacunar infarction: MR imaging. Radiology 1988;169: 101-04 CrossRef Medline

15. Song CJ, Kim JH, Kier EL, et al. MR imaging and histologic features of subinsular bright spots on T2-weighted MR images: VirchowRobin spaces of the extreme capsule and insular cortex. Radiology 2000;214:671-77 CrossRef Medline
16. Ramirez J, Berezuk C, McNeely AA, et al. Visible Virchow-Robin spaces on magnetic resonance imaging of Alzheimer's disease patients and normal elderly from the Sunnybrook Dementia Study. $J$ Alzheimers Dis 2015;43:415-24 CrossRef Medline

17. Hiroki M, Miyashita K, Oda M. Tortuosity of the white matter medullary arterioles is related to the severity of hypertension. Cerebrovasc Dis 2002;13:242-50 CrossRef Medline

18. Roher AE, Kuo YM, Esh C, et al. Cortical and leptomeningeal cerebrovascular amyloid and white matter pathology in Alzheimer's disease. Mol Med 2003;9:112-22 Medline

19. Doubal FN, MacLullich AM, Ferguson KJ, et al. Enlarged perivascular spaces on MRI are a feature of cerebral small vessel disease. Stroke 2010;41:450-54 CrossRef Medline

20. Wang R, Fratiglioni L, Laveskog A, et al. Do cardiovascular risk factors explain the link between white matter hyperintensities and brain volumes in old age? A population-based study. Eur J Neurol 2014;21:1076-82 CrossRef Medline

21. Rollins NK, Deline C, Morriss MC. Prevalence and clinical significance of dilated Virchow-Robin spaces in childhood. Radiology 1993;189:53-57 CrossRef Medline

22. Scheltens $P$, Barkhof $F$, Leys $D$, et al. A semiquantative rating scale for the assessment of signal hyperintensities on magnetic resonance imaging. J Neurol Sci 1993;114:7-12 CrossRef Medline

23. Wahlund LO, Barkhof F, Fazekas F, et al; European Task Force on Age-Related White Matter Changes. A new rating scale for age-related white matter changes applicable to MRI and CT. Stroke 2001; 32:1318-22 CrossRef Medline

24. Achiron A, Faibel M. Sandlike appearance of Virchow-Robin spaces in early multiple sclerosis: a novel neuroradiologic marker. AJNR Am J Neuroradiol 2002;23:376-80 Medline

25. Salzman KL, Osborn AG, House P, et al. Giant tumefactive perivascular spaces. AJNR Am J Neuroradiol 2005;26:298-305 Medline

26. Selvarajah J, Scott M, Stivaros S, et al. Potential surrogate markers of cerebral microvascular angiopathy in asymptomatic subjects at risk of stroke. Eur Radiol 2009;19:1011-18 CrossRef Medline

27. Tarnaris A, Tamangani J, Fayeye O, et al. Virchow-Robin spaces in idiopathic normal pressure hydrocephalus: a surrogate imaging marker for coexisting microvascular disease? Acta Neurochir Suppl 2012;113:33-37 CrossRef Medline

28. Chen W, Song X, Zhang Y. Assessment of the Virchow-Robin spaces in Alzheimer disease, mild cognitive impairment, and normal aging, using high-field MR imaging. AJNR Am J Neuroradiol 2011;32: 1490-95 CrossRef Medline

29. Gutierrez J, Rundek T, Ekind MS, et al. Perivascular spaces are associated with atherosclerosis: an insight from the Northern Manhattan Study. AJNR Am J Neuroradiol 2013;34:1711-16 CrossRef Medline

30. Yakushiji Y, Charidimou A, Hara M, et al. Topography and associations of perivascular spaces in healthy adults: the Kashima scan study. Neurology 2014;83:2116-23 CrossRef Medline

31. Shimada K, Kawamoto A, Matsubayashi K, et al. Silent cerebrovascular disease in the elderly: correlation with ambulatory pressure. Hypertension 1990;16:692-99 CrossRef Medline

32. Osborn AG, Preece MT. Intracranial cysts: radiologic-pathologic correlation and imaging approach. Radiology 2006;239:650-64 CrossRef Medline

33. Hansen TP, Cain J, Thomas O, et al. Dilated perivascular spaces in the basal ganglia are a biomarker of small-vessel disease in a very elderly population with dementia. AJNR Am J Neuroradiol 2015;36: 893-98 CrossRef Medline

34. Hernández Mdel C, Piper RJ, Wang X, et al. Towards the automatic computational assessment of enlarged perivascular spaces on brain magnetic resonance images: a systematic review. J Magn Reson Imaging 2013;38:774-85 CrossRef Medline

35. Wang X, Valdés Hernández Mdel C, Doubal F, et al. Development and initial evaluation of a semi-automatic approach to assess perivascular spaces on conventional magnetic resonance images. J Neurosci Methods 2016;257:34-44 CrossRef Medline 